\title{
The good and bad of microglia/macrophages: new hope in stroke therapeutics
}

\author{
Ya-chi HUANG, Zhong-ping FENG*
}

Acta Pharmacologica Sinica (2013) 34: 6-7; doi: 10.1038/aps.2012.178; published online 24 Dec 2012

$I^{s}$ schemic stroke contributes up to $80 \%$ of total stroke incidence and is one of the leading causes of death and disability worldwide ${ }^{[1,2]}$. Noteworthily, the inflammatory responses triggered after ischemic stroke often amplify and exacerbate the ischemic lesion ${ }^{[2]}$. Insufficient blood supply to brain cells initiates the pathophysiology of ischemic stroke and results in a densely ischemic core surrounded by a poorly perfused region termed ischemic penumbra. Cells within the ischemic area become deprived of oxygen, glucose, and other nutrients. Since oxygen and glucose are critical fuels for brain cell survival, lack of these factors leads to dysfunction of neurons, glial cells, endothelial cells, and pericytes. Neuronal microenvironments are no longer tightly maintained and disruption of the blood brain barrier (BBB) occurs. Blood borne immune cells start to infiltrate through the disrupted vessel walls into the ischemic area and release inflammatory mediators, which exaggerate brain edema and promote cell death in the penumbra to result in secondary infarct lesion ${ }^{[1,3]}$. Accordingly, ischemic stoke patients who arrived to the hospital within a short time window $\left(3-4.5 \mathrm{~h}^{[1,}\right.$ ${ }^{2,4]}$ ) after stroke incidence are commonly treated with the thrombolytic agent,

Department of Physiology, Faculty of Medicine, University of Toronto, 1 King's College Circle, Toronto, Ontario, Canada M5S $1 A 8$ *Correspondence to: Prof Zhong-ping FENG (zp.feng@utoronto.ca) recombinant tissue plasminogen activator (tPA, commonly called "clot buster"), in an attempt to restore perfusion and minimize brain damage ${ }^{[1,2]}$. For patients who have missed the short time frame, there is no safe and efficacious treatment that is currently available ${ }^{[3]}$. This is because the thrombus in the occluded artery has become too firm for tPA to dissolve. Moreover, the BBB disruption is likely to occur at this point, which would allow tPA to gain excess into the extravascular compartment. tPA residing in the extravascular compartment is associated with higher risk of intracranial hemorrhage and neurotoxicity ${ }^{[5]}$. Therefore, the beneficial effect of tPA is greatly compromised in patients with prolonged and untreated ischemic stroke. To develop effective treatments for these patients, it is essential to understand the complex and incompletely defined post ischemic inflammatory response mechanisms. This article highlights a recent work reported by $\mathrm{Hu}$ and colleagues $^{[6]}$, which sheds new light into the future management of post-ischemic stroke inflammation.

Resident microglia and peripheral macrophages play pivotal roles in the post-ischemic inflammation ${ }^{[7]}$. Activation of these cells after ischemia is known to produce detrimental inflammatory factors that are harmful to the surrounding tissues ${ }^{[7]}$. In this study, $\mathrm{Hu}$ and colleagues demonstrated that the microglia/macrophages actually play dual and opposite roles in the inflammatory responses progression in stroke $^{[6]}$. Microglia/macrophage initially differentiate into a neuroprotective phenotype termed M2, and then gradually transform into a neuronal injurious phenotype known as the M1 phenotype. M1 and M2 phenotypes are activated by different factors. M1 designates macrophages that are "classically activated via toll-like receptors or interferon- $\gamma$ ", whereas M2 denotes macrophages that are "alternatively activated by interleukin 4 or interleukin 13" ${ }^{\text {[7] }}$ M1-differentiated macrophages produce high levels of oxidative metabolites ( $e g$, nitric oxide and superoxide) and pro-inflammatory cytokines, which are essential for host defense and tumor cell killing, and cause collateral damage to healthy cells/tissues. On the other hand, M2 microglia/ macrophages promote angiogenesis and matrix remodeling while suppressing destructive immunity ${ }^{[7]}$.

$\mathrm{Hu}$ et al reported that injured neurons tend to prime microglia toward M1 phenotype ${ }^{[6]}$. Their in vitro experiments revealed that resting microglia began to express M1 markers, when they were treated with a conditioned culture media, in which neurons underwent oxygen glucose deprivation (OGD). OGD is an in vitro simulation of hypoxic/anoxic injury of neurons. These M1-like microglia not only exhibited the reduced phagocytosis, but also produced more inflammatory mediators. Whereas M1 macrophages are neural destructive, M2 macrophages play a neu- 
ral protective role to the ischemic brain area. Interestingly, when co-cultured with OGD-treated neurons, microglia were induced into M1 phenotype, and the M1 microglia only exacerbated death of OGD neurons, not OGD-untreated neurons. On the contrary, pre-differentiated M2 microglia/macrophages exerted protective effects to the OGDtreated neurons and revealed no specific changes to healthy neurons. In line with the finding above, culture media containing inflammatory factors released by M1 and M2 microglia was demonstrated differential effects on OGD-treated neurons. That is, conditioned culture media favoring M1 microglia differentiation promoted OGD-treated neuron demise, whereas conditioned culture media inducing M2 microglia differentiation granted neuroprotective effects on OGD- treated neurons. Both media did not cause significant changes to healthy neurons, which were not exposed to OGD conditions. Thus, the authors described the dual role of M1 and M2 microglia/ microphages in hypoxic/anoxic neurons in vitro.

In conclusion, $\mathrm{Hu}$ and colleagues demonstrated that activation of microglia/macrophage by hypoxic/anoxic conditions to dual phenotypes is largely dependent on the temporal lesion environment in culture ${ }^{[6]}$. Microglia/ macrophages of the M2 phenotype were present at the initial stage of injury and exerted neuroprotective roles to prevent cell death, whereas the M1 phenotype at the later stage of injury is involved in neuronal damage (Figure 1). Future investigation identifying the mechanisms that promote macrophage dif-

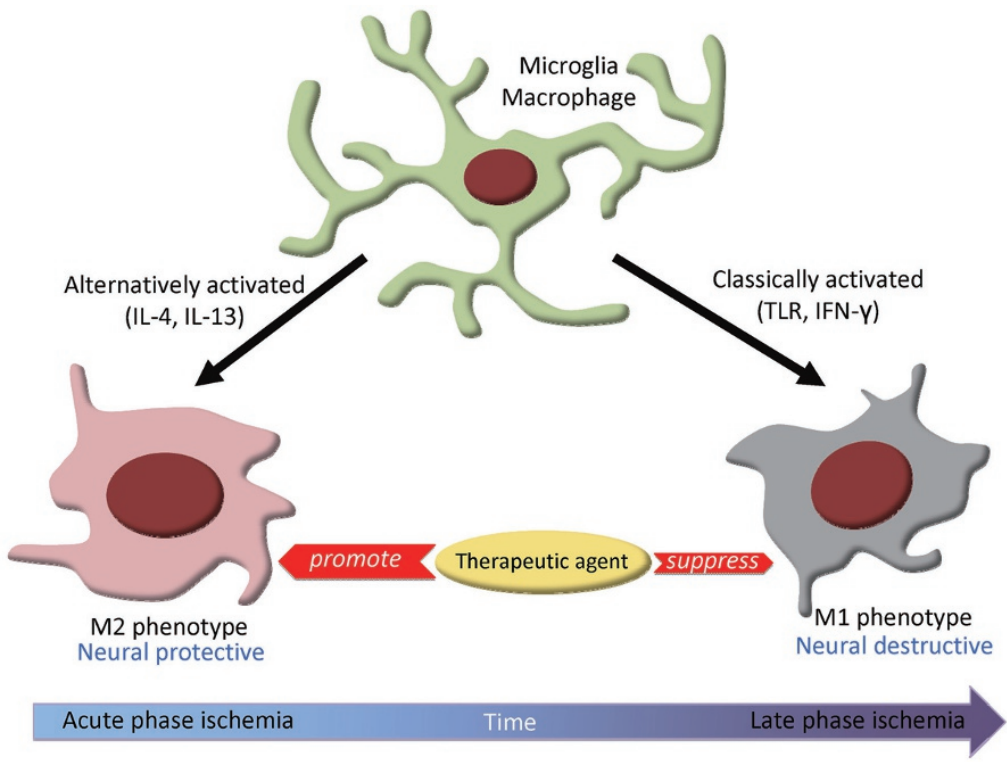

Figure 1. Microglia/macrophages possess dual but opposite roles in the inflammatory responses progression in stroke. At early stage of hypoxic/anoxic condition, microglia/macrophages are mostly differentiated into the neuroprotective M2 phenotype. With time, microglia/macrophages gradually transform into a neuronal injurious M1 phenotype. M1 phenotype is "classically activated via tolllike receptors or interferon-y", whereas M2 phenotype is "alternatively activated by interleukin 4 or interleukin 13". Future investigation identifying new therapeutic agents that promote macrophage differentiation into M2 phenotype and suppress transformation of macrophage into M1 phenotype would be valuable in treating post-ischemic stroke inflammation. (IL-4, interleukin 4; IL-13, interleukin 13; TLR, toll-like receptor; IFN-y, interferon-y) ferentiation into M2 phenotype can be valuable for developing new drugs for post-ischemic stroke inflammation. However, the major challenge in this field is to test whether the neuroprotective role of M2 activation at the initial stage is also seen in in vivo stroke model in animals. While this study provided the additional evidence indicating the yin and yang faces of the plastic microglia/macrophages in neuronal damage, a number of critical questions remain to be addressed. For instance, what are the factors in the hypoxic/anoxic environment that signals the differentiation of macrophage toward M1 and M2 phenotypes? M1 macrophage is neural destructive, why ischemic neurons favor the M1 macrophage phenotype? Finally and fundamentally, do the signaling mechanisms seen in culture trigger the similar responses in microglia/ macrophage following ischemic stroke in vivo in animals and in patients? Nevertheless, this study initiates the new approaches for future stroke treatment.
1 Shichita T, Ago T, Kamouchi M, Kitazono T, Yoshimura A, Ooboshi H. Novel therapeutic strategies targeting innate immune responses and early inflammation after stroke. J Neurochem 2012; 123 Suppl 2: 29-38.

2 Moskowitz MA, Lo EH, ladecola C. The science of stroke: mechanisms in search of treatments. Neuron 2010; 67: 181-98.

3 Sughrue ME, Mehra A, Connolly ES Jr, D’Ambrosio AL. Anti-adhesion molecule strategies as potential neuroprotective agents in cerebral ischemia: a critical review of the literature. Inflamm Res 2004; 53 : 497-508.

4 Marler JR, Goldstein LB. Medicine. Stroke - tPA and the clinic. Science 2003; 301: 1677.

5 Kaur J, Zhao Z, Klein GM, Lo EH, Buchan AM. The neurotoxicity of tissue plasminogen activator? J Cereb Blood Flow Metab 2004; 24: 945-63.

$6 \mathrm{Hu}$ X, Li P, Guo Y, Wang H, Leak RK, Chen S, et al. Microglia/Macrophage polarization dynamics reveal novel mechanism of injury expansion after focal cerebral ischemia. Stroke 2012; 43: 3063-70.

7 Perry VH, Nicoll JA, Holmes C. Microglia in neurodegenerative disease. Nat Rev Neurol 2010; 6: 193-201. 